\title{
Research on Early Warning Model of College Students' Psychological Crisis Based on Genetic BP Neural Network
}

\author{
Jia Wang ${ }^{1,}$, Zijie Zhang ${ }^{1}$, Haiji Luo ${ }^{1}$, Yinghao Liu ${ }^{1}$, Wei Chen ${ }^{1}$, Gang Wei ${ }^{2}$ \\ ${ }^{1}$ College of Medical Informatics, Chongqing Medical University, Chongqing, China \\ ${ }^{2}$ College of Electrical Engineering, Chongqing University of Science Technology, Chongqing, China

\section{Email address:} \\ 102509@cqmu.edu.cn (Jia Wang),17754925898@163.com (Zijie Zhang),18875044211@163.com (Haiji Luo), \\ 13983808487@163.com (Yinghao Liu),18883850965@163.com (Wei Chen), eecqwei@163.com (Gang Wei) \\ *Corresponding author
}

\section{To cite this article:}

Jia Wang, Zijie Zhang, Haiji Luo, Yinghao Liu, Wei Chen, Gang Wei. Research on Early Warning Model of College Students' Psychological Crisis Based on Genetic BP Neural Network. American Journal of Applied Psychology. Vol. 8, No. 6, 2019, pp. 112-120.

doi: 10.11648/j.ajap.20190806.12

Received: October 26, 2019; Accepted: November 18, 2019; Published: November 26, 2019

\begin{abstract}
With the continuous advancement of information construction in colleges and universities, a large number of student data have been accumulated and precipitated in the campus center database of colleges and universities. On the basis of social constructivist psychology, Maslow and Mittelmann's mental health standards and related research results of psychological crisis early warning, three first-level indicators and 15 second-level indicators of college student's psychological crisis were established. The campus data of 1504 college students were collected on one data center of a college, and the weight of each indicator was determined on the basis of correlation analysis of each indicator and psychological status indicators through SPSS21.0 and the expert opinion. The early warning model of college students' psychological crisis was basically constructed. With experimental simulation of 250 sets of real data, the early warning model based on the genetic BP Neural network for its initial weight and threshold with MATLAB was improved. The results indicated that the indicator system of college student's psychological crisis in this paper was effective and feasible, and the early warning model based on genetic BP neural network had high accuracy and certain application value.
\end{abstract}

Keywords: College Student, Psychological Crisis Early Warning Model, Genetic BP Neural Network

\section{Introduction}

In recent years, with the increasing pressure of studies, employment and interpersonal relationship, psychological the frequent emergence of college students' crisis incidents brings bad social impacts to not only students, but also families, colleges, universities, and the government. Most psychological crises have signs before they break out, accompanied by obvious emotional changes, such as anxiety, fear, excitement, despondency, depression, etc. and the individual's changes in campus life. At present, college psychological status is generally investigated by means of regular questionnaires. It is difficult to accurately grasp the traces of psychological crisis of a large number of college students on campus and capture the real psychological status of students. Constructing an on-field accurate psychological crisis early warning system to catch the symptoms of crisis, analyze and control the influencing factors of psychological crisis on time will effectively reduce the occurrence of psychological crisis in colleges and universities.

\section{Literature Review of Psychological Early Warning System}

Since G. Caplanh first put forward the theory of psychological crisis in 1964, many scholars have carried a lot of research on college students' psychological crisis by employing scale measurement statistics [1], behavior of warning index [2-3], crisis theory [4], retrospective study [5], education and training of crisis management [6], emergency assessment, physical and physiological assessment, psychological assessment and psychological crisis level 
assessment [7]. Some exploratory work has been carried out in the study of psychological prediction by using neural networks. Pourshahriar compares the effects of Logistic Regression (LR) and Artificial Neural Networks (ANNs) in the application of psychology [8]. Shan constructs a warning and evaluation model of post-disaster psychological crisis based on principal component analysis and neural network, providing faster and more effective prediction and management methods [9]. These researches show that the neural network is effective and feasible for psychological prediction. However, the fact that BP neural network is prone to lead the algorithm falling into local extremum will create the failure of network training. In order to solve this problem, scholars try to optimize BP neural network through genetic algorithm, and verify it in the aspects of academic performance prediction [10-11] and athlete performance prediction [12]. Experiments prove that, the genetic BP neural network will get more accurate training results.

\section{The Construction of Early Warning System for College Students' Psychological Crisis}

The early warning index system of psychological crisis is the basis of the psychological crisis management, analysis and early warning for college students. Psychological status is not the presentation of a single psychological factor, and the outbreak of psychological crisis is inextricably linked with various social factors. Maslow and Mittelmann put forward ten mental health standards around sense of security, self-understanding, realistic environment, personality, learning, emotional control, personality and personal needs, emphasizing the importance of environment, collective life and social requirements for individual mental health, etc. Vygorsky put forward Social Constructivist Psychology and pointed out that human psychology developed in social activities [13]. Scholars have explored early warning indicators based on emotions, learning, interpersonal communication, physiology, family background, social environment, individual development status, stressors, stress response, individual crisis susceptibility factors, etc.[14-16]. However, they mainly focus on qualitative analysis with a lack of quantitative criteria for indicators and operability, and it brings considerable difficulties to the prevention and treatment of college students' psychological crisis.

With the continuous advancement of information construction in colleges and universities, a large number of student data have been accumulated and precipitated in the campus center database of colleges and universities. Although It involves in a wide range of real data, it has not been deeply excavated and utilized. On the basis of extensive reference to Social Constructivist Psychology, Maslow's and Mittelmann's mental health standards and related research results on early warning of psychological crisis, 3 first-level early warning indicators and 15 second-level early warning indicators of college students' psychological crisis, namely, academic situation data, life situation data and social situation data, were drawn up. In this study, campus data of 1504 college students were collected from the central database of a university in Chongqing from 2018 to 2019, and 15 kinds of data, such as school, life, social communication, etc., were extracted as full sample data and the data were correlated with college students' psychological evaluation data through SPSS21.0 for the weight set of each indicator.

\subsection{Construction of Weight of Various Indicators Based on Fuzzy Comprehensive Evaluation}

On fully consideration of the characteristics of interaction among different college students' campus situations, five college teachers with psychological health consultation background of more than five years were invited to make a general evaluation of the college students' campus psychological situation and the restrictive factors of their mental health, and determine the first-level indicators and second-level indicators. Referring to the membership theory of Fuzzy Mathematics, 15 second-level indicators were selected, as quantitative evaluation factors, and the quantitative indicators were graded and distributed in the interval of $[0.1,0.9]$.

The correlation between various indicators and psychological status indicators were tested through SPSS21.0. With the combination of experts' opinion, the weights of indicators at all levels were drawn up, and the following assumptions were made on their nature, as shown in Table 1:

Table 1. Weights of the First Level and Second Level Indicators of the Psychological Status of College Students.

\begin{tabular}{llll}
\hline $\begin{array}{l}\text { First level } \\
\text { indicator }\end{array}$ & Second level indicator & $\begin{array}{l}\text { Training } \\
\text { weight }\end{array}$ & number \\
\hline \multirow{3}{*}{ Social situation } & cadre status & 0.5 & $\mathrm{a}$ \\
& political status & 0.3 & $\mathrm{~b}$ \\
& holiday retention & 0.2 & $\mathrm{c}$ \\
& absenteeism & 0.2 & $\mathrm{~d}$ \\
Academic & GPA & 0.1 & $\mathrm{e}$ \\
situation & performance & 0.1 & $\mathrm{f}$ \\
& body test results & 0.1 & $\mathrm{~g}$ \\
& scholarships & 0.1 & $\mathrm{~h}$ \\
& enrollment transaction & 0.3 & $\mathrm{~m}$ \\
& fail situation & 0.1 & $\mathrm{i}$ \\
& subsidies & 0.2 & $\mathrm{j}$ \\
Life situation & dormitory cleaning & 0.15 & $\mathrm{k}$ \\
& daily violations & 0.2 & 1 \\
& region & 0.15 & $\mathrm{n}$ \\
& Family & 0.3 & $\mathrm{o}$ \\
\hline
\end{tabular}

\subsection{The Construction of the Early Warning Level of College Students' Psychological Crisis on Campus}

In order to present the results visually, based on the test results of psychological state, 4 levels of psychological crisis early warning, namely, no warning, slight warning, moderate warning and severe warning, are constructed. The data in $[0,1]$ interval are used to represent four expected outputs, as shown in Table 2: 
Table 2. Warning Level of College Students' Psychological Crisis.

\begin{tabular}{ll}
\hline Psychological crisis early warning level & expected output \\
\hline health, no warning & $(0.7,0.9]$ \\
slight problem, slight warning & $(0.5,0.7]$ \\
moderate problem, moderate warning & $(0.3,0.5]$ \\
serious problem, severe warning & $(0.1,0.3]$ \\
\hline
\end{tabular}

\subsection{The Construction of Psychological Crisis Prediction Warning System for College Students}

Based on all kinds of indicators of fuzzy comprehensive evaluation and prediction warning level of psychological crisis, the prediction warning system of psychological crisis of college students was constructed as shown in Figure 1:

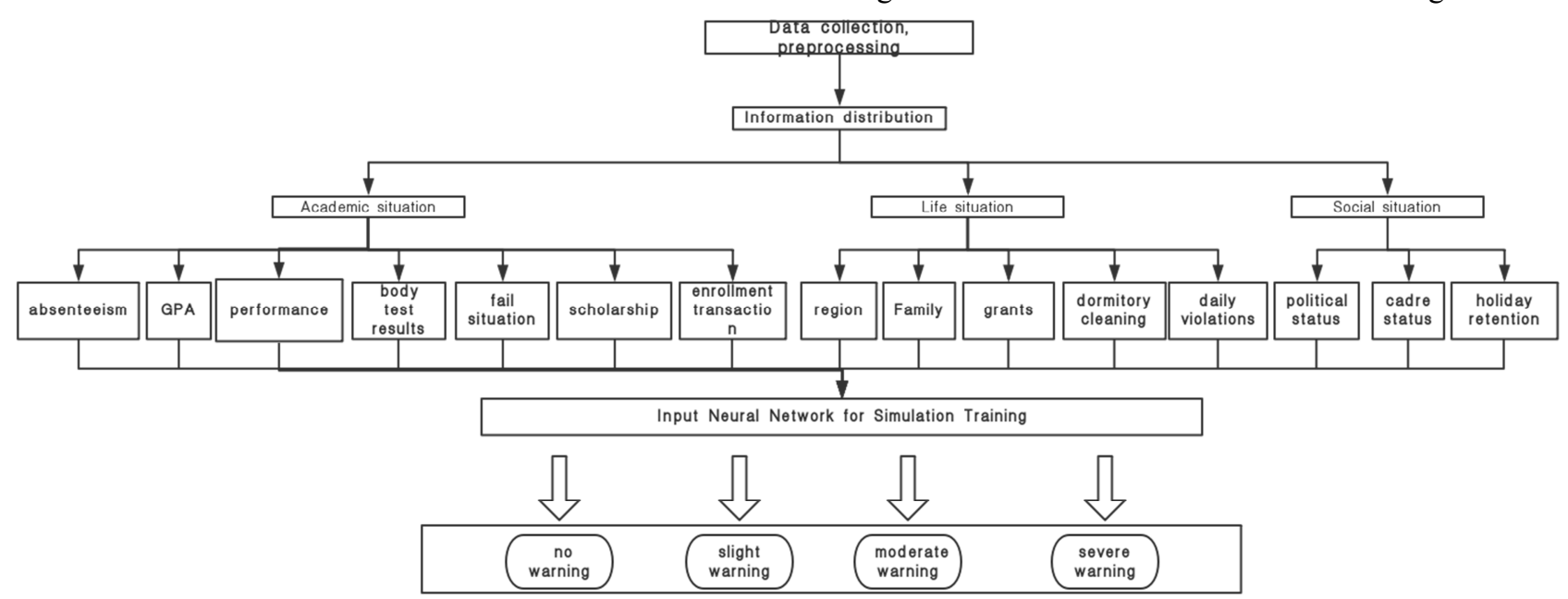

Figure 1. Prediction Warning System of College Students' Psychological Crisis.

Among which:

Absenteeism/GPA/ Fail refers to students absenteeism / grade point average/fail in the compulsory subjects.

Scholarship refers to state-level/provincial-ministerial/school-level scholarship awarded to students.

Student status change refers to students' demotion/ suspension/transfer or other change.

Source area/family situation refers to students' source of origin/economic situation of family.

Grants refers to all kinds of state-level/provincial-ministerial/school-level grants received by students.

Cadre status refers to students' cadres status at all levels of school/college/class.

Stay in school on holidays refers to the retention of students during holidays (not winter and summer holidays).

\section{Optimizing BP Neural Network Model by Genetic Algorithms}

\subsection{BP Neural Network}

BP (Back propagation) Neural Network, a local search optimization method with nonlinear mapping capability, self-learning and self-adapting abilities, can deal well with the problem of non-linear system. However, it also has some inherent limitations and problems. During the error back propagation process, the change of its weights was continuous without mutation when optimizing the weights of nodes. In such a change, it is easy for the weight to converge to a special value--local minimum, and difficult to come to the next minimum. If the gradient does not change significantly in these multiple iterations, the network training will be ended with a sub-optimal solution instead of the global optimal solution, resulting in unsatisfactory training effects.

\subsubsection{Transfer Function}

Activation function plays an important role in neural network. Due to its monotone increasing and inverse function monotone increasing, Sigmoid function was chosen as the threshold function of the neural network in this study. Variables were mapped between $0-1$, defined by the formula as follows:

Unipolar S-type function

$$
S(x)=\frac{1}{1+e^{-x}}
$$

\subsubsection{Normalization}

Since a large dimension difference exists between psychological early warning indicators, and the input value of S-type activation function needs to be controlled in $[0,1]$ and $[-1,1]$, in this study, the input data of BP neural network was processed by linear normalization to ensure that the data changes in a group of samples in the maximized state in the range of $[0,1]$, shown as the following formula:

$$
X_{j}^{\prime}=\frac{\left(X_{j}-X_{j \min }\right)}{X_{j \max }-X_{j \min }}
$$

Among them, $X_{j \min }, X_{j \max }$ were the maximum value and minimum value of $X_{j}$.

\subsection{Genetic Algorithms}

Genetic Algorithms (GA), a global parallel stochastic search optimization algorithm on the basis of biological mechanisms of natural selection and genetic variation, codes a 
random solution of the problem as a "chromosome" to calculate its fitness, transfer the parameters by the selection, crossover and mutation of operators, and find the optimal solution by screening the fitness values. With the characteristics of self-organization, self-adaptability and uncertainty, genetic algorithm is able to automatically acquire and accumulate the feature characteristics about search space in the search process, control the search process self-adaptively, reserve the expected data, and carry out the next cycle. The optimization by genetic algorithm will avoid the problem of local minimum of BP neural network, so as to achieve a better training effect.

\subsubsection{Fitness Function}

Fitness is the pros and cons of individual. When genetic algorithm is employed to optimize the BP neural network, the initial weights and thresholds of the network will be optimized effectively to reduce the prediction error of the network. According to the difference between the actual output and the expected output of the network, the fitness function formula was selected as follows:

$$
\mathrm{E}=\sum_{\mathrm{i}=1}^{\mathrm{n}}\left|T_{i}-Y_{i}\right|^{2}
$$

In the above formula, $n$ is the number of output nodes, $T_{i}$ and $Y_{i}$ are the actual output and expected output of the training sample $i$ of the network. In order to find a smaller error, the reciprocal formula was chosen as reciprocal:

$$
f=\frac{1}{E}
$$

\subsubsection{Selection Operator}

The selection probability of operator is proportioned to individual fitness value. The greater the fitness of an individual is, the greater probability to be selected, and the greater probability for the individual to be inherited.

Providing the fitness of the $i$ individual was $f_{i}$, then the probability $P_{s i}$ that the individual $i$ being reserved will be:

$$
P_{s i}=\frac{f_{i}}{\sum_{j=1}^{n} f_{j}}
$$

\subsubsection{Crossover Operator}

Generated by the crossover operation of $X_{1}$ and $X_{2}$, $X_{1}^{\prime}$ and $X_{2}^{\prime}$ represent two new individuals gene codes during crossover of the father generation as follows:

$$
\left\{\begin{array}{l}
X_{1}^{\prime}=\alpha X_{1}+(1-\alpha) X_{2} \\
X_{2}^{\prime}=\alpha X_{2}+(1-\alpha) X_{1}
\end{array}\right.
$$

In this formula, $\alpha$ is combination coefficient. There are two crossover modes in the crossover process: partial crossover mode which uses different values of $\alpha$ and overall crossover mode which uses the same values of $\alpha$. The overall crossover mode was adopted in this study, with the formula as follows:

$$
a=\left\{\begin{array}{lc}
0.6 & f_{i}<f_{\text {avg }} \\
\frac{f_{\max }-f_{i}}{f_{\max }-f_{\text {avg }}} & f_{i} \geq f_{\text {avg }}
\end{array}\right.
$$

In the above formula, $f_{i}$ represented the fitness value of the individual $i$ in the father generation, $f_{\max }$ represented the maximum fitness value of the individual $i$ in the father generation, and $f_{\text {avg }}$ represented the average fitness value of the individual $i$ in the father generation.

\subsubsection{Mutation Operator}

The mutation operator can guarantee the population diversity of genetic algorithm and the random searching ability of the algorithm. The variant model of this case is as follows:

$$
\begin{gathered}
f(g)=\left[r \times\left(1-\frac{g}{m}\right)\right]^{2} \\
\left\{\begin{array}{l}
c^{\prime}=c+(c-1) \times f(g) \\
c^{\prime}=c+(1-c) \times f(g)
\end{array}\right.
\end{gathered}
$$

$r$ is a random number, $g$ is the current number of iterations, $m$ is the maximum number of iterations, $c$ is the random position on the chromosome.

\subsection{Optimizing BP Neural Network by Genetic Algorithms}

At present, three methods are popularly used in optimizing genetic BP Neural network [17]:

(1) to change the initial weights and thresholds;

(2) to learn the topological structure of network;

(3) to improve the learning rules of network.

In this study, genetic algorithm was used to optimize the initial weights and thresholds of BP neural network, increase the probability of the optimal solution being selected, and find the optimal initial weights of BP neural network through code, selection, crossover and mutation operation. Combined with the non-linear fitted ability of BP neural network and the powerful searching ability of optimal solution of genetic algorithm, it would have a better prediction of the function output.

\section{Early Warning Modeling and Simulation of College Students' Psychological Crisis}

Based on the early warning indicators, the early warning model of psychological crisis based on BP neural network and that optimized by genetic BP neural network were built respectively, and the results of simulation and test data of the two kinds of early warning networks were analyzed and compared. 


\subsection{Early Warning Modeling and Simulation of College Students' Psychological Crisis Based on BP Neural Network}

\subsubsection{Training Sample Data}

In this study, 250 sets of basic data were selected as the sample data of the network, among which 200 sets $(80 \%)$ were taken as the training samples of the network, and 50 sets (20\%) were taken as the test samples of the network. Due to space limitations, only four sets of pre-treated training samples were listed in this study, as shown in Table 3.

Table 3. Training Sample.

\begin{tabular}{|c|c|c|c|c|c|c|c|c|c|c|c|c|c|c|c|c|}
\hline \multirow{2}{*}{ number } & \multicolumn{15}{|c|}{ Pretreatment data } & \multirow{2}{*}{$\begin{array}{l}\text { expected } \\
\text { output }\end{array}$} \\
\hline & $\mathbf{a}$ & b & c & d & e & f & g & h & i & $\mathbf{j}$ & $\mathbf{k}$ & 1 & $\mathbf{m}$ & $\mathbf{n}$ & o & \\
\hline 1 & 0.45 & 0.15 & 0.135 & 0.27 & 0 & 0.359 & 7 & 0.05 & 0 & 0.18 & 0.01 & 0.105 & 0 & 0.27 & 0.02 & 0.5 \\
\hline 2 & 0.25 & 0.27 & 0.075 & 0.15 & 0 & 0.262 & 9.7 & 0.09 & 0 & 0.1 & 0.01 & 0.075 & 0 & 0.27 & 0.02 & 0.7 \\
\hline 3 & 0.05 & 0.15 & 0.075 & 0.27 & 0.12 & 0.08 & 8.7 & 0.05 & 1.3 & 0.18 & 0.01 & 0.075 & 0 & 0.27 & 0.02 & 0.3 \\
\hline 4 & 0.25 & 0.1 & 0.135 & 0.27 & 0 & 0.314 & 7 & 0.05 & 0 & 0.18 & 0.01 & $0 . .75$ & 0.2 & 0.27 & 0.02 & 0.9 \\
\hline
\end{tabular}

\subsubsection{Determination of the Number of Neurons in the Hidden Layer}

There are two ways to improve the network accuracy: increasing the number of neurons in the hidden layer and increasing the number neurons in the hidden layer. In this study, we chose to increase the number of neurons in the hidden layer to improve the accuracy.

The specific number of neurons in the hidden layer was determined by the input and output of the network. The formula was as follows:

$$
m=\sqrt{n+l}+a
$$

$m$ was the number of nodes in the hidden layer, $n$ was the number of nodes in the input layer, $l$ was the number of nodes in the output layer, and a is a constant between 1 to 10 .

In this study, the input nodes were 15 . By using the FAC function, the input nodes were reduced to 4 and output node was 1 after dimensionality reduction. The selection range of neurons in the hidden layer could be estimated from the formula, and the number of neurons could be determined through trial and error comparison in training. On the premise of ensured the network generalization ability, less number of neurons would be chosen.

In this network, the number of input neurons was $n=4$, the number of output neurons was $i=1$, and the training accuracy of the network was set to 0.001 . The number of neuron in the hidden layer was selected in the range of $[3,13]$. The test results of training errors were shown in Table 4:

Table 4. Comparison of Training Errors for Different Hidden Layers.

\begin{tabular}{lll}
\hline $\begin{array}{l}\text { number of neurons in } \\
\text { the hidden layer }\end{array}$ & error precision & training time \\
\hline 3 & 0.1256 & 7 \\
4 & 0.11273 & 7.4 \\
5 & 0.09683 & 7.9 \\
6 & 0.07941 & 9.5 \\
7 & 0.06514 & 10.1 \\
8 & 0.05317 & 10.2 \\
9 & 0.04578 & 10.9 \\
10 & 0.04075 & 12.3 \\
11 & 0.03487 & 12.8 \\
12 & 0.0271 & 13 \\
13 & 0.02703 & 13.6 \\
14 & 0.02907 & 11.34 \\
\hline
\end{tabular}

As shown in Table 4, when the number of hidden layer neurons is "12" or "13", the training error value was lower than 0.028 , which could meet the requirements of high precision. While the timeliness of "12"was higher than that, the error rebound occurred when the number of hidden layer neurons was "14", accord with the empirical formula (10). Since this experimental data belonged to the small sample training, it did not consider the training timeliness, after a comprehensive comparison, this study selected "12" to be the number of hidden layer neurons.

\subsubsection{Topological Structure Diagram of BP Neural Network}

In this study, a two-layer neural network structure was adopted. The numbers of neurons in the input layer and the output layer were set to 4 and 1 respectively, the number of neurons in the hidden layer was set to 12 , the transfer function between the input layer and the hidden layer was S-type function, and the transfer function between the hidden layer and the output layer was Tansig function. The final BP neural network topology was shown in Figure 2.

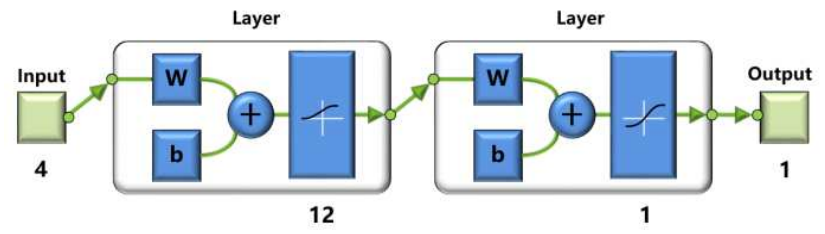

Figure 2. Topological Structure Diagram of BP Neural Network.

The parameters of BP neural network were designed as follows: the network topology was 4-12-1; the maximum number of training was 5000; the convergence accuracy was 0.01 ; the learning rate was 0.01 , the initial weight threshold was a random number between $[-1,1]$, the transferred function gain $\mathrm{a}=1$, confirming that the validation check was 6 .

\subsection{Early Warning Modeling and Simulation of Psychological Crisis for College Students Based on Genetic BP Neural Networks}

Based on the same parameters of BP neural network, the genetic algorithm parameters were set by multiple tests from the selection of smaller mean square errors as follows: The population size was set to a scale, the crossover probability 
was set to 0.6 , variation probability was 0.2 , the maximum number of iterations was 50. The optimal individual was optimized by genetic algorithm, the individual was decoded, the decoded weights and thresholds were used as the initial weights and thresholds of BP Neural Network, and the network simulation training was carried out. The simulation training steps were shown in Figure 3.

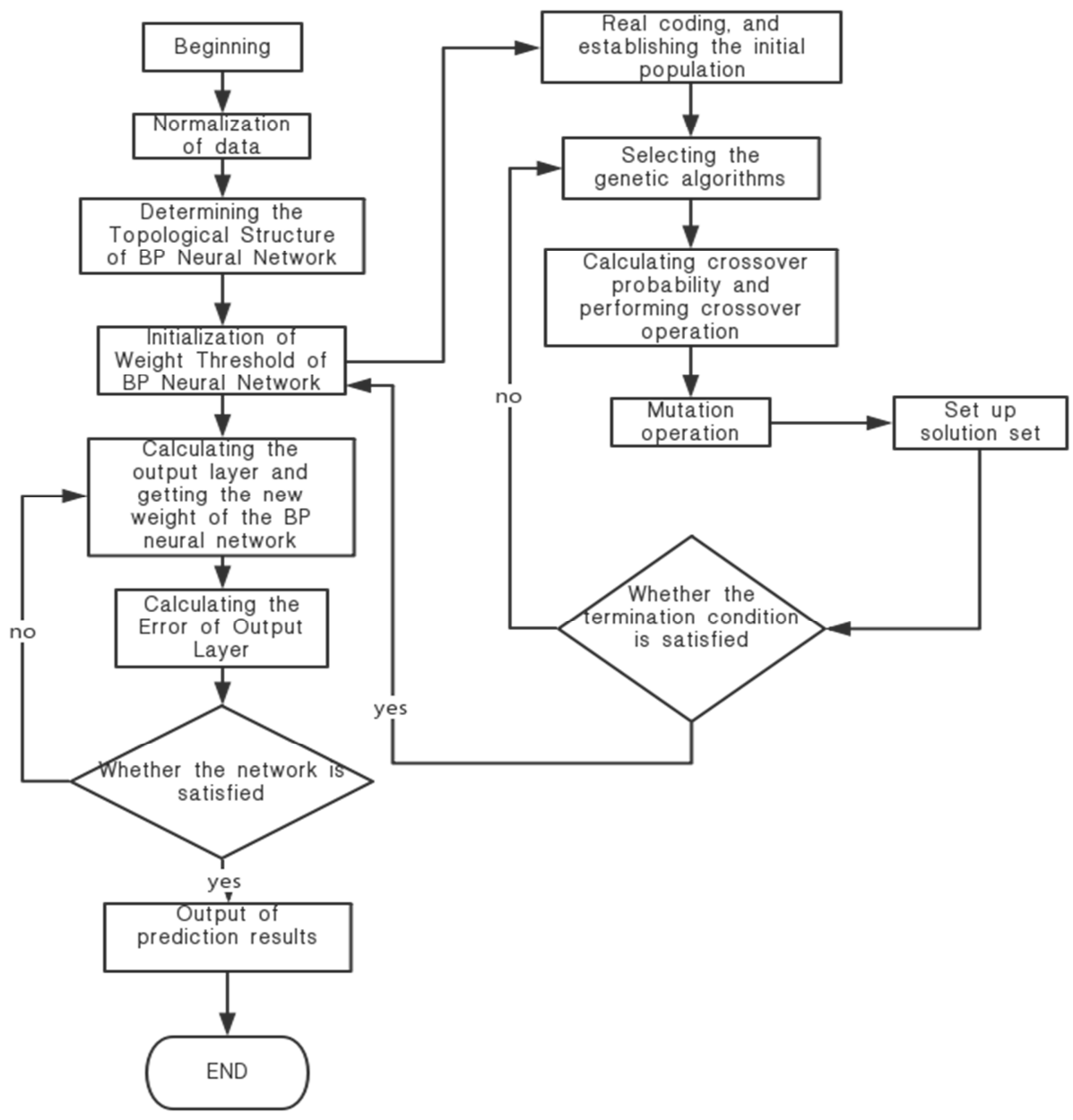

Figure 3. Simulation Steps of Genetic BP Neural Network.

In the MATLAB environment, the same 250 sets of sample data including 200 training sets and 50 testing sets were trained. Through the network operation, the predicted value and real value of 50 testing sets of genetic BP neural network were compared with that of the BP neural network, as shown in Figure 4:

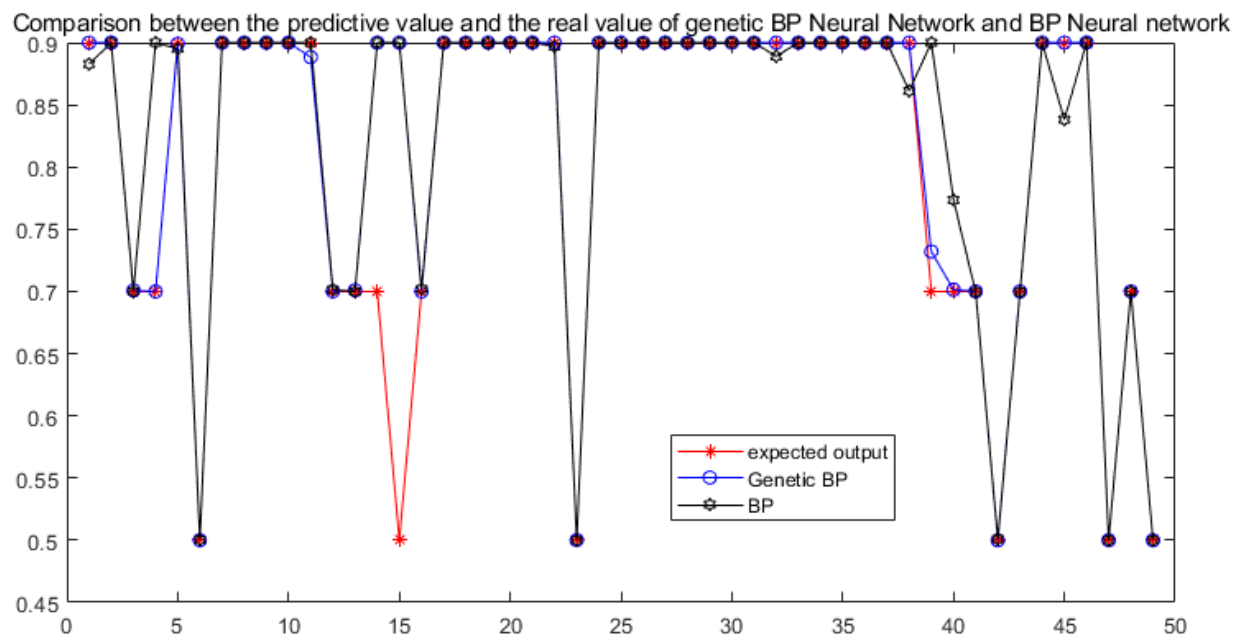

Figure 4. Comparison between the Predictive Value and the Real Value of Genetic BP Neural Network and BP Neural network. 
In the prediction of 50 test sets, the prediction error curves of genetic BP neural network and neural network were shown in Figure 5:

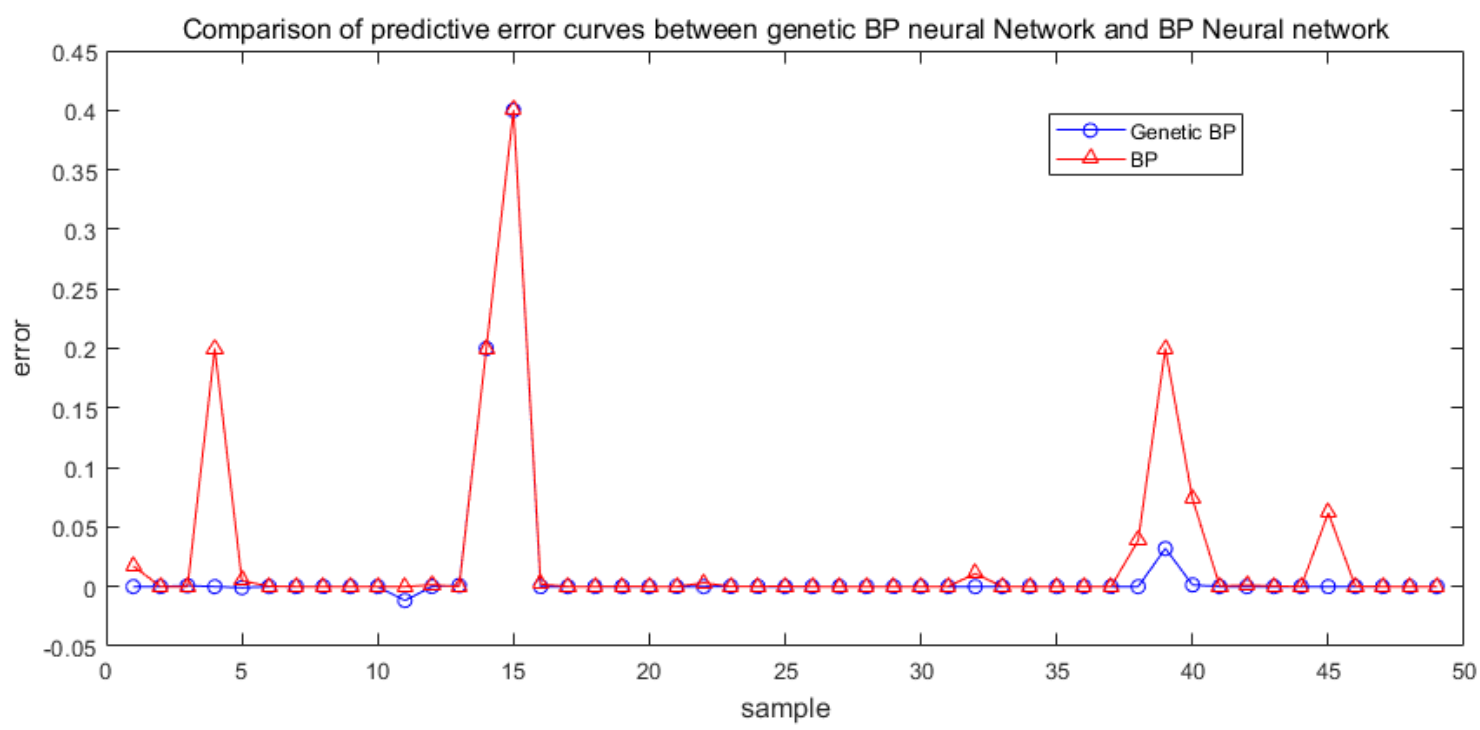

Figure 5. Comparison of Predictive Error Curves between Genetic BP Neural Network and BP Neural Network.

In this study, the expected value of training error was set to be 0.1 . When the absolute error exceeded 0.1 , the diagnostic error occurred. After BP neural network was trapped in the local optimal solution, the prediction error curves of genetic BP neural network and BP neural network were compared and shown in Figure 6.

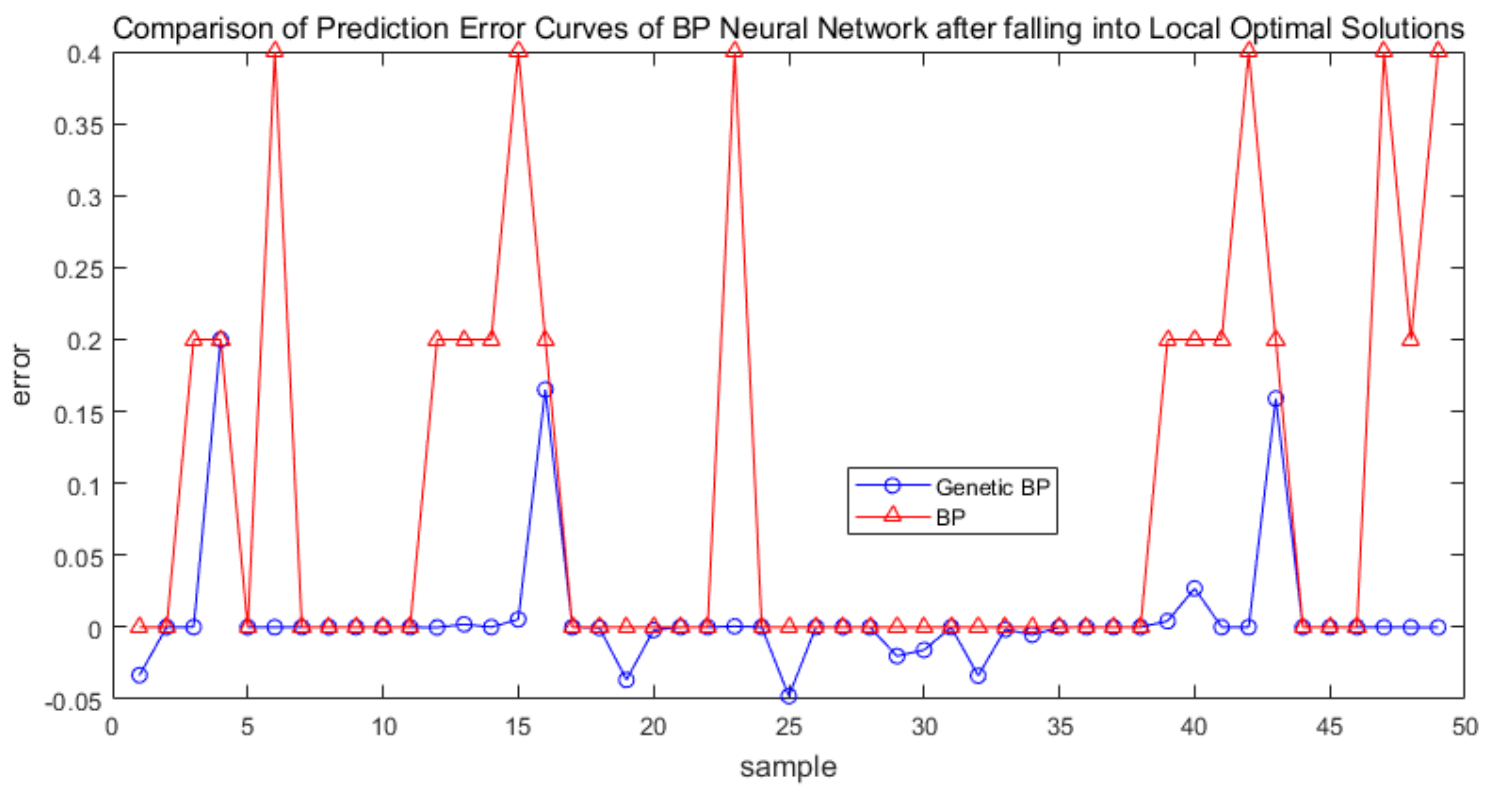

Figure 6. Comparison of Prediction Error Curves of BP Neural Network after Falling into Local Optimal Solutions.

Table 5. Simulation Training Results of Early warning Model of College Students'Psychological Crisis.

\begin{tabular}{|c|c|c|c|c|}
\hline Serial number & Expected output & Training output & Alert level & warning accuracy \\
\hline 1 & 0.9 & 0.90000 & health, no warning & Yes \\
\hline 2 & 0.7 & 0.69872 & slight problem, slight warning & Yes \\
\hline 3 & 0.5 & 0.50012 & moderate problem, moderate warning & Yes \\
\hline 4 & 0.3 & 0.30002 & serious problem, severe warning & Yes \\
\hline
\end{tabular}

From the above analysis, the following conclusions could be obtained:

(1) The training error based on BP neural network could not basically satisfy the set expectation value, and there was a big error in the diagnosis of test samples. The maximum absolute error reached 0.4 , the average absolute error reached 0.0286 with large error fluctuation. For the test of 50 sets of test samples, the 
accuracy rate was only $82 \%$.

(2) The training error of genetic BP Neural network could satisfy most of the set expectations. The average absolute error of 50 test samples was $0.0150,47.55 \%$ lower than that of BP neural network, and the diagnostic accuracy of test samples was increased to $96 \%$.

(3) The reasons for the errors in the samples might include the following reasons: the training samples were not large enough, there might be erroneous data in the samples, the BP neural network was prone to over-fitting, and the generalization ability was insufficient.

\section{Conclusion and Expectation}

Based on the campus situations of college students, this study established an early warning model of college students 'psychological crisis on the basis of genetic BP Neural network, and drew the following conclusions:

(1) College students' psychological status was complex, and it was easy to be influenced by many kinds of stressors. There were many kinds of factors related to psychological crisis and the ways of stress were complex. Based on the study, life and social data of college students' on campus, the first-level and second-level indicators related to psychological crisis were constructed, and a number of indicators were classified and data preprocessed to truly reflect the status of influencing factors of college students' psychological situation.

(2) With powerful abilities of nonlinear mapping ability, self-learning and parallel processing, BP neural network processed the psychological crisis early warning by classification. In view of the defects of slow convergence sped and inclination to fall into local flow minimum, genetic algorithm was adopted to optimize BP neural network. By optimizing the initial threshold of weights of the neural network, it could effectively avoid the network falling into local minimum and achieved a better training effect.

(3) The results of 200 sets of data and the results of 50 sets of data showed that the genetic BP neural network improved greatly in convergence speed, test error and diagnostic accuracy of the network. The results of early warning was practical, and it was suitable as an early warning method for college students psychological crisis.

In order to promote the accuracy of early warning and improve the early warning system of psychological crisis, it is necessary for a further research mainly focusing on the following issues:

(1) To collect more data of college students from different regions, universities and levels, improving the samples of psychological crisis, and enhancing the universal significance and popularity of the early warning model.

(2) To further optimize the algorithm aiming at the local search ability, searching speed and stability.
(3) To build dynamic early warning model of college students' psychological crisis, and realize an on-field online early warning of college students' psychological crisis.

\section{Acknowledgements}

Thanks are due to Han Baoru and Jia Yuanyuan for valuable discussion.

My paper is supported by the Scientific and Technological Research Program of Chongqing Municipal Education Commission, China (General program). Grant No. 17SKG026.

\section{References}

[1] Pei Tao, Zhou Hong, Chen Yu, et al. Study on the Applied of Early Warning of Mental Crisis in Institutions of Higher Education [J]. College Counselors, 2015, 12 (6): 21-25.

[2] Lu Jun, Xu Jianmei. Behavior Observational Warning Indicator System for Psychological Disorder "Gatekeepers" among College Students [J]. Forecast and Analysis, 2016, 5: 94-99.

[3] Yang Xueling, Zhang Pining, Liang Ting, et al. A Study on the Early warning Index of Mental Disorder Gatekeeper Behavior in College Students [J]. Modern Preventive Medicine, 2016, 43 (7): 1246-1249.

[4] Holahan C J, Moos R H. Life Stressors, Resistance Factors, and Improved Psychological Functioning [J]. Journal of Personality \& Social Psychology, 1990, 58 (5): 909-17.

[5] Jollant F, Lawrence N L, Olié, Emilie, et al. The Suicidal Mind and Brain: A Review of Neuropsychological and Neuroimaging Studies [J]. World Journal of Biological Psychiatry, 2011, 12 (5): 319-339.

[6] Ye Huasong. A Study on the Management System of Psychological Crisis Intervention for College Students [J]. Research on Higher Education in Heilongjiang Province, 2008, 5: 141-143.

[7] Wu Caihong. Risk Assessment and Crisis Intervention of Mental Health of College Students [J]. Journal of Hunan Institute of Finance and Economics, 2011, 4 (27): 143-145.

[8] Pourshahriar, H. Correct vs. Accurate Prediction: A Comparison between Prediction Power of Artificial Neural Networks and Logistic Regression in Psychological Researches [J]. 4th International Conference of Cognitive Science, 2012, 32: 97-103.

[9] Shan W, Zhou Q, Pan Y, et al. Forewarning Assessment of Psychological Crisis in Post-disaster Based on, Principal Component Analysis and Neural Network [C]. International Conference on Biomedical Engineering \& Informatics. IEEE, 2010 .

[10] Luo Yongguo. Improved Genetic Algorithm Based Student Score Prediction Model [J]. Bulletin of Science and Technology, 2012, 10 (28): 223-225.

[11] Chen Yong. Research and implementation of result prediction based on genetic neural network [J]. Modern Electronics Technique, 2016, 39 (5): 96-100. 
[12] Chen Haiying, Guo Qiao, XU Li. Prediction Method of 100 Sprint Performance Based on Hybrid Genetic Neural Network [J]. Computer Simulation, 2004, 2 (21): 89-91.

[13] Vygowski. Educational Psychology (Chinese Edition) [M]. Hangzhou: Zhejiang Education Press, 2001.

[14] Gu Guangling, Mao Dequan, Xu Zhenghong, Shi Yuqin. Thoughts on Constructing the Early warning System of College Students' Psychological Crisis [J]. Journal of Chuzhou University, 2006, 6: 108-109.

[15] Guo Lan, Fu Anzhou, Huo Shaozhou. Research on
Psychological Crisis and Early warning System of College Students [J]. Journal of China University of Geosciences (Social Science Edition), 2001, 3: 63-67.

[16] Chen Xiang, Jia Jucai. A Study on College Students' Psychological Crisis and its Pre-warning Mechanism [J]. Educational Exploration, 2005, 6: 110-111.

[17] Sun Lingfang, Zhou Jiapo, Lin Weijian, etc. On Network Public Opinion Crisis Early Warning Based on the BP Neural Network and Genetic Algorithm [J]. Intelligence Journal, 2014, 11, 33 (11): 18-24. 